\title{
Evidence of environmental dependencies of Type la supernovae from the Nearby Supernova Factory indicated by local $\mathrm{H} \alpha$ (Corrigendum)
}

\author{
M. Rigault ${ }^{1}$, Y. Copin ${ }^{1}$, G. Aldering ${ }^{2}$, P. Antilogus ${ }^{3}$, C. Aragon ${ }^{2}$, S. Bailey ${ }^{2}$, C. Baltay ${ }^{4}$, S. Bongard ${ }^{3}$, C. Buton ${ }^{5}$, \\ A. Canto ${ }^{3}$, F. Cellier-Holzem ${ }^{3}$, M. Childress ${ }^{6}$, N. Chotard ${ }^{1}$, H. K. Fakhouri ${ }^{2,7}$, U. Feindt ${ }^{5}$, M. Fleury ${ }^{3}$, \\ E. Gangler ${ }^{1}$, P. Greskovic ${ }^{5}$, J. Guy ${ }^{3}$, A. G. Kim ${ }^{2}$, M. Kowalski ${ }^{5}$, S. Lombardo ${ }^{5}$, J. Nordin ${ }^{2,8}$, P. Nugent ${ }^{9,10}$, \\ R. Pain $^{3}$, E. Pécontal ${ }^{11}$, R. Pereira ${ }^{1}$, S. Perlmutter ${ }^{2,7}$, D. Rabinowitz ${ }^{4}$, K. Runge ${ }^{2}$, C. Saunders ${ }^{2}$, \\ R. Scalzo ${ }^{6}$, G. Smadja ${ }^{1}$, C. Tao ${ }^{12,13}$, R. C. Thomas ${ }^{9}$, and B. A. Weaver ${ }^{14}$ (The Nearby Supernova Factory) \\ ${ }^{1}$ Université de Lyon, Université de Lyon 1, CNRS/IN2P3, Institut de Physique Nucléaire de Lyon, 69622 Lyon, France \\ e-mail: mickael.rigault@clermont.in2p3.fr \\ 2 Physics Division, Lawrence Berkeley National Laboratory, 1 Cyclotron Road, Berkeley, CA 94720, USA \\ ${ }^{3}$ Laboratoire de Physique Nucléaire et des Hautes Énergies, Université Pierre et Marie Curie Paris 6, \\ Université Paris Diderot Paris 7, CNRS-IN2P3, 4 place Jussieu, 75252 Paris Cedex 05, France \\ ${ }^{4}$ Department of Physics, Yale University, New Haven, CT 06520-8121, USA \\ ${ }^{5}$ Physikalisches Institut, Universität Bonn, Nußallee 12, 53115 Bonn, Germany \\ ${ }^{6}$ Research School of Astronomy and Astrophysics, The Australian National University, Mount Stromlo Observatory, Cotter Road, \\ Weston Creek, ACT 2611, Australia \\ 7 Department of Physics, University of California Berkeley, 366 LeConte Hall MC 7300, Berkeley, CA 94720-7300, USA \\ ${ }^{8}$ Space Sciences Laboratory, University of California Berkeley, 7 Gauss Way, Berkeley, CA 94720, USA \\ ${ }^{9}$ Computational Cosmology Center, Computational Research Division, Lawrence Berkeley National Laboratory, \\ 1 Cyclotron Road MS 50B-4206, Berkeley, CA 94611, USA \\ ${ }^{10}$ Department of Astronomy, University of California Berkeley, B-20 Hearst Field Annex \# 3411, Berkeley, CA 94720-34110, USA \\ ${ }^{11}$ Centre de Recherche Astronomique de Lyon, Université Lyon 1, 9 avenue Charles André, 69561 Saint Genis Laval Cedex, France \\ 12 Tsinghua Center for Astrophysics, Tsinghua University, Beijing 100084, PR China \\ ${ }^{13}$ Centre de Physique des Particules de Marseille, 163 avenue de Luminy, Case 902, 13288 Marseille Cedex 09, France \\ ${ }^{14}$ Center for Cosmology and Particle Physics, New York University, 4 Washington Place, New York, NY 10003, USA
}

A\&A, 560, A66 (2013), https://doi.org/10.1051/0004-6361/201322104

Key words. cosmology: observations - errata, addenda

While undertaking the next phase of analysis of the correlation of Type Ia supernova (SN Ia) brightnesses with properties of their local host environments, we discovered a software error affecting the measurements used in Rigault et al. (2013). The measurements of host-galaxy $\mathrm{H} \alpha$ emission, $\Sigma_{\mathrm{H} \alpha}$, and resulting local star formation rate, LSFR, measured for the environment surrounding each SN in Rigault et al. (2013) where not in units of $M_{\odot} \mathrm{yr}^{-1} \mathrm{kpc}^{-2}$, as intended, but rather in units of $M_{\odot} \mathrm{yr}^{-1} \operatorname{arcsec}^{-2}$ averaged over a $1 \mathrm{kpc}$ aperture. Here we provide corrected values for several key quantities, finding that these do not significantly change the main conclusions of the original paper.

At our typical redshift of $z \sim 0.05$, the $1 \mathrm{kpc}$ aperture used in Rigault et al. (2013) has a projected angular size of $\sim 1$ arcsec, so the overall scale of the measurements is unchanged. Measurements for SNe Ia spanning our lowest to highest redshift, i.e., $0.03<z<0.08$, have scale ranges from 1.65 arcsec per kpc to 0.66 arcsec per kpc, changing $\Sigma_{\mathrm{H} \alpha}$ by at most \pm 0.4 dex at the redshift extremes and producing an RMS change of 0.2 dex. This is to be compared to the nearly 3 dex range spanned by $\Sigma_{\mathrm{H} \alpha}$.

In Rigault et al. (2013) the sample was divided evenly between SNe Ia in local environments above (Ia $\alpha$ ) or below (Ia $\epsilon$ ) $\log \left(\Sigma_{\mathrm{H} \alpha}\right)=38.35$ dex. There we further identified two grouping of SNe Ia: $M_{1}$ were those fainter than average and having
$\Sigma_{\mathrm{H} \alpha}$ values spanning the full observed range, while $M_{2}$ were those brighter and having $\Sigma_{\mathrm{H} \alpha}$ values below our $\Sigma_{\mathrm{H} \alpha}$ threshold. With the corrections here, this boundary shifts by -0.03 dex, to $\log \left(\Sigma_{\mathrm{H} \alpha}\right)=38.32$ dex.

While the boundary barely moves, some SNe Ia cross the boundary. In particular, two overly bright $\mathrm{SNe}$ Ia formerly assigned to the Ia $\epsilon$ group move into the Ia $\alpha$ group. These SNe Ia were part of a group of three cases discussed in Sect. 7.3 of Rigault et al. (2013), where we explored the impact of moving the $\Sigma_{\mathrm{H} \alpha}$ threshold and the potential impact of misassociations for $\mathrm{Ia} \epsilon$ with the Ia $\alpha$ group due to boosting by the warm interstellar medium (WIM) or accidental projection onto H II regions. One of these is just below our original inclination cut, reinforcing projection as an issue for this case. We originally decided that these SNe Ia belong to the $M_{2}$ group and they lie more than $4 \sigma$ from the $M_{1}$ group, so we retain them in the $M_{2}$ group even though their corrected $\Sigma_{\mathrm{H} \alpha}$ values are now slightly higher.

Figure 1 shows the distribution of Hubble residuals with the corrected $\Sigma_{\mathrm{H} \alpha}$ values, to be compared with the top and bottom panels of Fig. 6 in the original paper. The two SNe Ia mentioned above are highlighted using a lighter shading to fill their markers. It is apparent that the bimodality originally discovered in Rigault et al. (2013) is still present. The dispersion of the Ia $\alpha$ group remains tight, $0.114 \pm 0.013 \mathrm{mag}$, after rejecting the (now 3) 


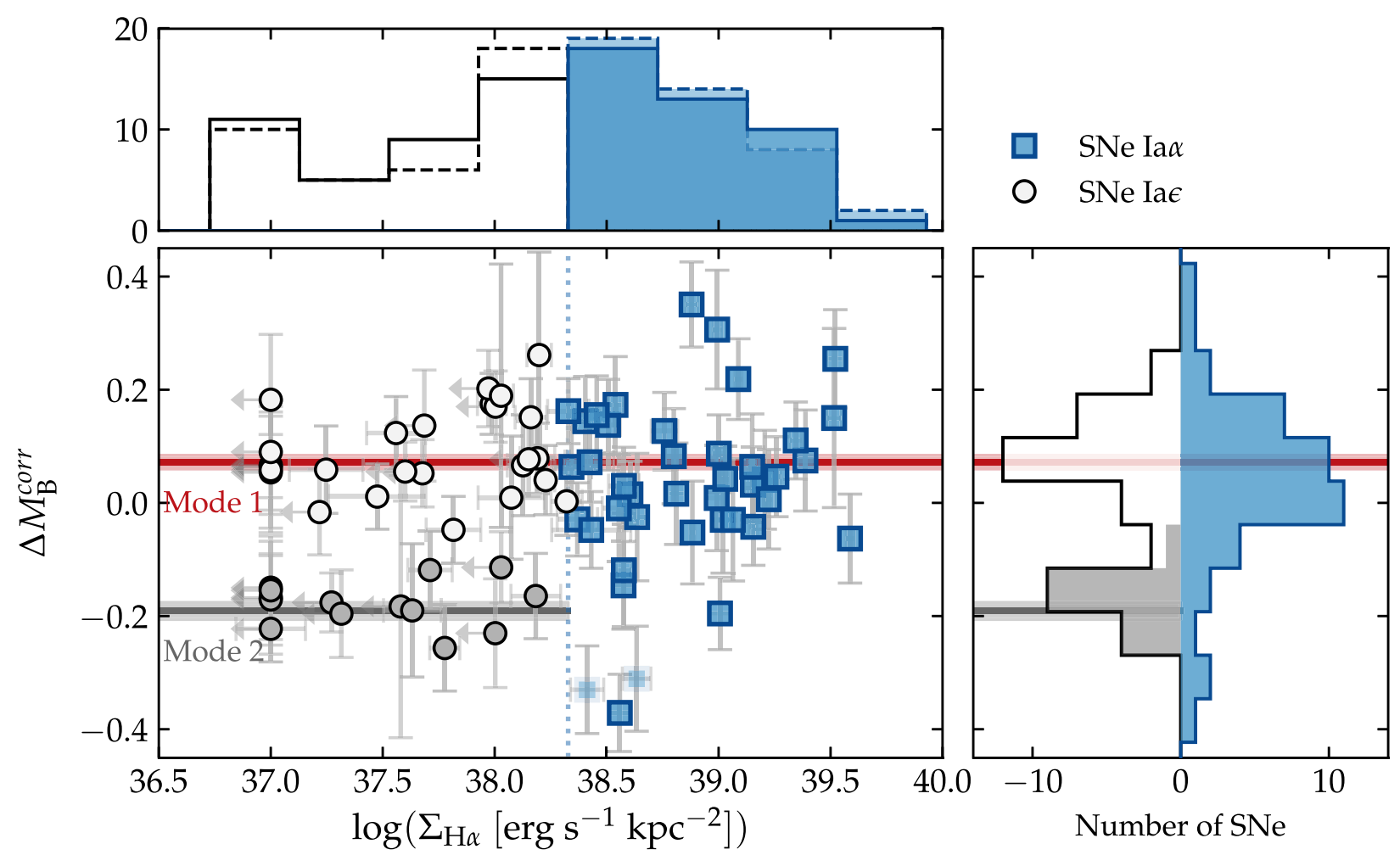

Fig. 1. Hubble residuals versus the now-corrected $\Sigma_{\mathrm{H} \alpha}$ within $1 \mathrm{kpc}$ surrounding each SN Ia. Squares represent Ia $\alpha$ SNe Ia, while circles represent those categorized as Ia $\epsilon$. Among the Ia $\epsilon$, those with lighter gray belong to $M_{1}$, while those with darker gray are in $M_{2}$. The two SNe Ia that are overly bright and that have now moved above the SFR threshold (vertical dashed line) are shaded light blue. The original means of the $M_{1}$ and $M_{2}$ subsamples are shown as horizontal lines, and labeled accordingly. It can be seen that the two modes have moved slightly closer together. The upper panel shows the $\Sigma_{\mathrm{H} \alpha}$ histograms for the corrected (solid line) and the original (dashed line) values.

bright $\mathrm{SNe} \mathrm{Ia} \alpha$, and the dispersion of the Ia $\epsilon$ group remains larger, at $0.143 \pm 0.016 \mathrm{mag}$. The difference in standardized magnitudes now has a step of $0.063 \pm 0.029$ mag between SNe Ia in the Ia $\alpha$ and Ia $\epsilon$ groups, within the error bar of the original $0.094 \pm 0.031 \mathrm{mag}$. The offset between the means of the $M_{1}$ and $M_{2}$ samples is $0.260 \pm 0.020 \mathrm{mag}$, compared to the original $0.262 \pm 0.019 \mathrm{mag}$. As these values are essentially unchanged, our estimate for the evolution of the step in Hubble residuals with host galaxy mass and its implications for cosmology still hold. The correlations originally noted between $\Sigma_{\mathrm{H} \alpha}$ and the light curve stretch $(x 1)$ and color $(c)$ are also unaffected.

Subsequent to the analysis in Rigault et al. (2013) we have developed a more refined metric describing the local environments of SNe Ia (Rigault et al. 2015). This metric, the local specific star formation rate (LsSFR), more accurately separates these environment into younger and older categories. When applying this new indicator to the Rigault et al. (2013) dataset (as corrected here) the offset in brightness between SNe Ia from low and high LsSFR environments is $0.110 \pm$ $0.030 \mathrm{mag}$. For completeness, we also note that this error has no effect on the results of Rigault et al. (2015), which used GALEX data and separate computer code.

\section{References}

Rigault, M., Copin, Y., Aldering, G., et al. 2013, A\&A, 560, A66 Rigault, M., Aldering, G., Kowalski, M., et al. 2015, ApJ, 802, 20 